\title{
Performance limits of graphene-ribbon field-effect transistors
}

\author{
F. Muñoz-Rojas, ${ }^{1}$ J. Fernández-Rossier, ${ }^{1}$ L. Brey, ${ }^{2}$ and J. J. Palacios ${ }^{1,2}$ \\ ${ }^{1}$ Departamento de Física Aplicada, Universidad de Alicante, San Vicente del Raspeig, E-03690 Alicante, Spain \\ ${ }^{2}$ Instituto de Ciencia de Materiales de Madrid, Consejo Superior de Investigaciones Científicas, E-28049 Cantoblanco, Spain
}

(Received 5 September 2007; published 2 January 2008)

\begin{abstract}
The performance of field effect transistors based on an single graphene ribbon with a constriction and a single back gate are studied with the help of atomistic models. It is shown how this scheme, unlike that of traditional carbon-nanotube-based transistors, reduces the importance of the specifics of the chemical bonding to the metallic electrodes in favor of the carbon-based part of device. The ultimate performance limits are here studied for various constriction and metal-ribbon contact models. In particular, we show that, even for poorly contacting metals, properly tailored constrictions can give promising values for both the on conductance and the subthreshold swing.
\end{abstract}

DOI: 10.1103/PhysRevB.77.045301

PACS number(s): 73.23.-b, 73.22.-f, 73.63.-b

\section{INTRODUCTION}

A number of factors determining the performance of carbon nanotube field-effect transistors (CNTFET's) still remain to be mastered before these promising devices can compete with current Si-based transistors. One of these factors, probably the most important one, is the Schottky barrier formed at the interface between the source or drain metallic electrodes and the carbon nanotube. ${ }^{1-4}$ Current understanding attributes the different observed performances of CNTFET's to the different work function values of the metals used as electrodes. Furthermore, even for the same metal, a disparity of behaviors have been observed due to the difficulty in controlling the Schottky barrier, i.e., the resulting position of the Fermi energy $E_{F}$ with respect to the valence and conduction bands of the nanotube as a consequence of different fabrication schemes. These translate into a variety of barrier heights for holes or electrons and a variety of on currents and threshold voltages for either voltage gate polarity. ${ }^{1-4}$

A way to get around, the lack of reproducibility of current-voltage and transfer characteristics of CNTFET's is to avoid the use of metallic electrodes close to the semiconducting channel. This has been attempted by integrating Ohmic contacts in the carbon nanotube through a combination of back and top gates ${ }^{5}$ which allow for an independent control of the density on different regions of the nanotube. This scheme allows us to create metallic and semiconducting regions on a single nanotube. Band-gap engineering of graphene ribbons, ${ }^{6,7}$ on the other hand, offers an alternative strategy to the multiple-gate scheme. ${ }^{8,9}$ A single graphene ribbon with armchair edges and a narrow channel in the middle [see Figs. 1(a) and 1(c)] can present three regions with different band gaps which can be doped differently by a single back gate [see Fig. 1(b)]. For channel widths with a number a dimer lines $\neq 3 M-1$, with $M$ an integer smaller than $\approx 500$, the constriction presents a transport gap at room temperature ${ }^{10}$ and acts as the semiconductor active channel in standard transistors. Since the gap scales inversely with the width of the ribbon, the wide sections of the ribbon or leads can present a zero or vanishingly small gap for large enough widths and easily behave as metallic electrodes because of finite temperature or as they are driven out of the charge neutrality point by the action of the back gate voltage $V_{G}$. The same gate voltage controls the on-off state by bringing $E_{F}$ into the gap of the constriction or out of it [see Fig. $1(\mathrm{~b})]$.

Theoretically, this system should behave as an ambipolar transistor where the threshold voltage $V_{\text {th }}$ [the voltage at which the $I\left(V_{G}\right)$ characteristics begins to deviate from an exponential behavior] is determined solely by the width of the narrow channel. ${ }^{11}$ The on conductance, $G_{\text {on }}=d I /\left.d V\right|_{V_{G}>V_{\text {th }}}$, and the subthreshold swing, $S$ $=\left(d \log I / d V_{G}\right)^{-1}$, magnitudes that determine the transistor performance are also controlled by the capacitive couplings and by the atomic structure of the lead-channel contact. The ultimate performance limits of graphene ribbon field-effect

(a)

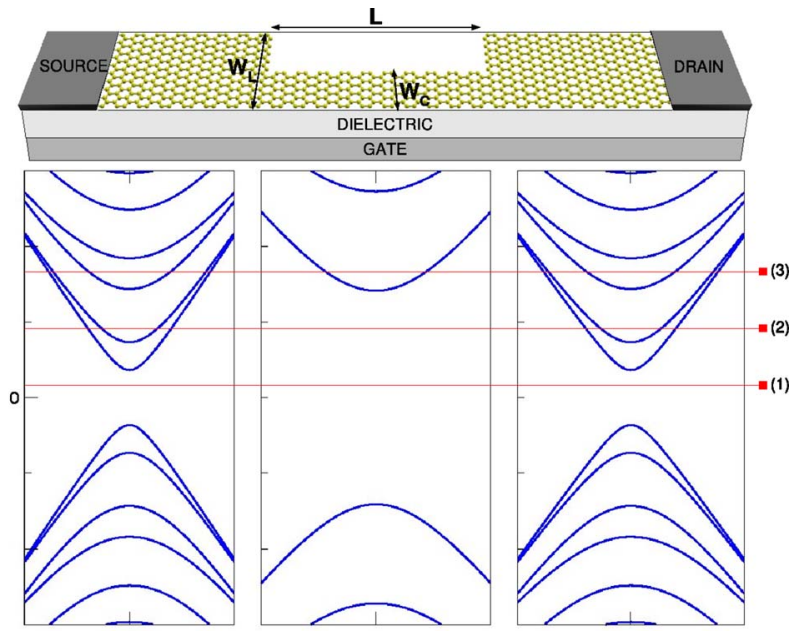

(c)

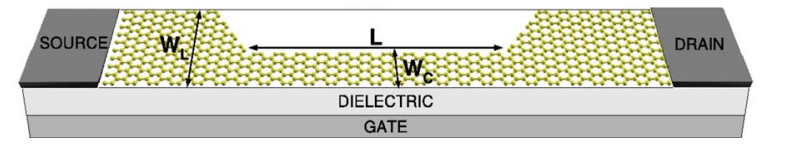

FIG. 1. (Color online) Schematic view of a graphene ribbon field-effect transistor with (a) square and (c) tapered constrictions placed on one side. (b) Example of band structures corresponding to the three regions: left lead, right lead, and constriction. The horizontal lines denote different Fermi levels corresponding to different conduction situations: (1) at the charge neutrality point, (2) off state, and (3) on state. 
transistors (GRFET's) are here established for two contact models. The first, square, where the crystallographic orientation of one edge changes by $90^{\circ}$ at the contact [see Fig. 1(a)]. The second, tapered, where the lead narrows down progressively until reaching the channel width. This is achieved in practice by a single change in the crystallographic orientation of one of the edges of $60^{\circ}$ [see Fig. 1(c)]. In this case, the armchair edge is never interrupted, except for the change in the crystallographic orientation. The channel is always placed laterally, i.e., on one side of the ribbon. This choice can be justified on the basis that in order to fabricate a constriction with reduced disorder, it is desirable to leave untouched one edge of the initial graphene layer or wide ribbon and, e.g., etch away only the other edge (constrictions created by etching both edges have already been fabricated ${ }^{8}$ ). Our proposed GRFET could simplify the fabrication process and might improve the final performance. A second justification will be mentioned when discussing the results.

\section{METHODOLOGY}

We compute the conductance of the ribbon with the constriction $G_{C}$ using the Landauer formalism which assumes coherent transport across the constriction. The electronic structure is calculated in the standard one-orbital tightbinding model $\mathrm{l}^{6,7,11-13}$ which has been shown to reproduce the low-energy physics of ribbons whose edges are saturated with hydrogen. The scattering problem is solved using the Green's function approach. This involves (i) the calculation of the Green's function projected on the constriction and on part of the left and right ribbon, (ii) the calculation of the self-energies for the semi-infinite ribbon leads by iterative solution of the Dyson equation, ${ }^{13}$ and (iii) the subsequent evaluation of the transmission probability $T_{C}$ using the Caroli expression. ${ }^{14}$ The details can be found in Ref. 13. In order to simplify the discussion, we assume first semi-infinite graphene ribbons on both sides. The role played by the metallic electrodes is considered at the end, although one can anticipate that they should not affect the results as long as $W_{L} \gg W_{C}$, where $W_{L}$ is the width of the bulk ribbon and $W_{C}$ is the width of the narrow channel.

\section{ZERO-TEMPERATURE RESULTS}

We begin by showing in Figs. 2 and 3 the zerotemperature $G_{C}$ for a channel with length $L \approx 10 \mathrm{~nm}$ and $W_{C}=1 / 2,1 / 4$, and $1 / 8 W_{L}$ for $W_{L}=5.8 \mathrm{~nm}$. For both types of contacts between leads and channel, we obtain the expected stepwise increase of the conductance as a function of $E_{F}$ (in units of the hopping parameter $t$ ), associated with the increase in the number of bands crossing $E_{F}$ in the channel. The steps come in pairs, reflecting the band structure of semiconducting armchair ribbons [see Fig. 1(b)]. On top of the steps, we obtain strong Fabry-Pérot-like oscillations as a consequence of the finite reflection at the channel-lead contacts. The periodicity of these oscillations is consistent with the length and with the dispersion relation in the channel. The amplitude of the oscillations, on the other hand, depends

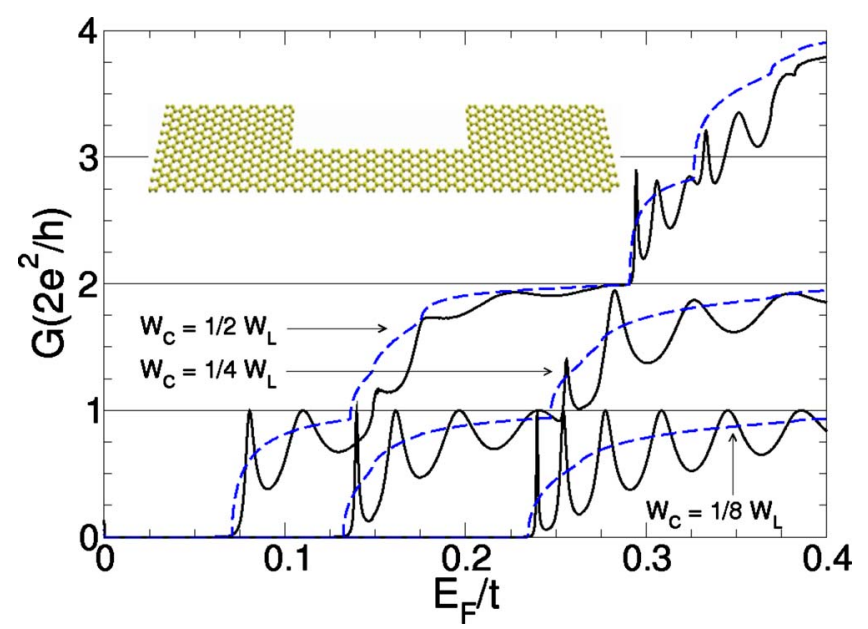

FIG. 2. (Color online) Solid lines correspond to the conductance of an $L \approx 10 \mathrm{~nm}$ channel for different widths in the case of the square contact model shown in the inset. The width of the lead is $W_{L}=5.8 \mathrm{~nm}$. In dashed lines, the conductance for a single interface is shown.

on how abrupt the contact is. For the square contact, the amplitude of the oscillations is larger than for the tapered one, as expected. In order to separate the contribution of the scattering at the interfaces from the quantum interference effects, we have also computed the transmission of a single interface, e.g., the transmission for a semi-infinite channel (dashed lines in Figs. 2 and 3). In all cases, the transmission steps saturate to their quantum limit very slowly. For both contact types, as the width of the channel decreases, the transmission worsens as a consequence of the increasing mismatch between lead and channel wave functions. The increasing reflection on decreasing $W_{C} / W_{L}$ is somewhat damped for the tapered contacts, but still clearly visible. When $W_{C} / W_{L} \rightarrow 0$, quasilocalized states form in the channel as a result of a significant loss in the transparency of the contacts. This situation resembles the formation of quantum dots in poorly contacted carbon nanotubes,${ }^{15}$ but without me-

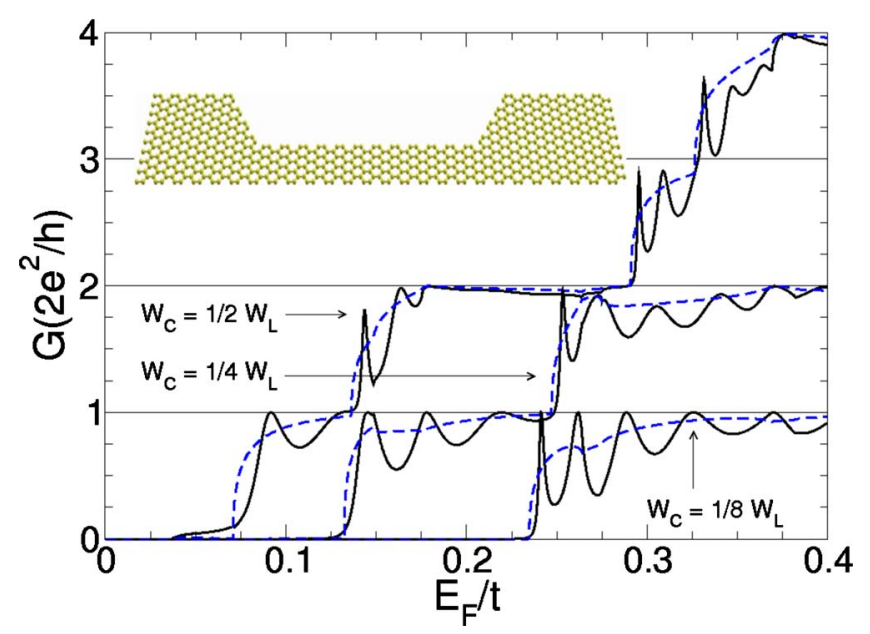

FIG. 3. (Color online) Same as in Fig. 2, but for the tapered contact model shown in the inset. 
tallic contacts. In general, one can anticipate that channels with tapered contacts to the leads should perform better than their square counterparts for both $G_{\text {on }}$ and $S$, although the overall performance diminishes as the channel narrows down in both models.

\section{ROOM-TEMPERATURE RESULTS}

We now turn our attention to the actual room-temperature performance of GRFET's. Although the channel widths and lengths considered $(\leqslant 10 \mathrm{~nm})$ are still difficult to achieve with present lithographic techniques, ${ }^{8}$ the results can be easily extrapolated to more realistic constrictions. The ones considered here behave as intrinsic semiconductors at room temperature since their gaps are in the range of $\approx 0.1-1 \mathrm{eV}$ for a typical value of $t=2.7 \mathrm{eV}$, which is much larger than $k T$ $\approx 25 \mathrm{meV}$ at room temperature. Figure 4 shows a logarithmic plot of $G\left(E_{F}\right)$ for all the cases previously studied. In all cases, the conductance oscillations have disappeared, smeared out by temperature. The combined action of finite reflection at the interfaces, finite temperature, and tunneling prevents $G_{\text {on }}$ from reaching the quantum limit $2 e^{2} / h$ for $E_{F}$ $>e V_{\text {th }}$ before the next channel opens up. We can also extract the respective values of $S$ from the logarithmic plots. While, for the wider channels, $S$ is larger for the tapered contacts ( 83 vs $66 \mathrm{meV} / \mathrm{dec}$ ade), these contacts outperform the square ones for the narrowest channels (63 vs $68 \mathrm{meV} /$ decade).

The above mentioned values for $S$ are lower limits to the ones expected in a real system where electrostatics play an important role. At the simplest level, ignoring changes in the electronic structure of the graphene system, the electrostatics can simply be taken into consideration through a functional

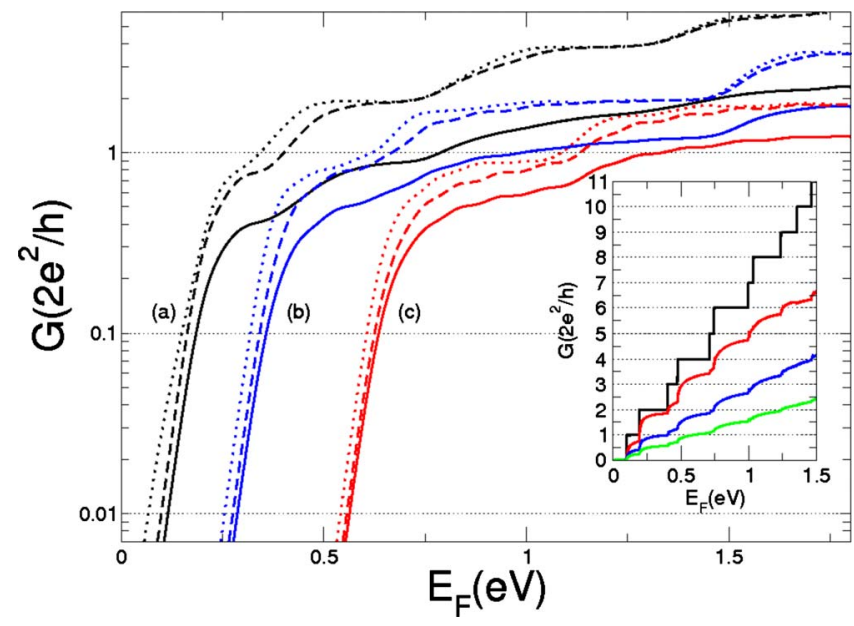

FIG. 4. (Color online) Logarithmic plot of the room-temperature conductance for the square (dashed lines) and tapered (dotted lines) contact models shown in Figs. 1(a) and 1(c) for different channel widths $W_{C} / W_{L}$ of (a) $1 / 2$, (b) $1 / 4$, and (c) $1 / 8$, with $W_{L}=5.8 \mathrm{~nm}$. For other parameters, see text. Solid lines denote the total conductance of the square model including metal electrodes with on-site energy $\epsilon=5 t$. The small panel shows the conductance of the perfect ribbon (solid line) and of the metal-ribbon interface for $\epsilon=0 t, 5 t$, and $10 t$ (top to bottom). relation between $E_{F}$ and $V_{G}$. This relation involves the dielectric constant $\epsilon$, the thickness of the dielectric spacer $d$, and the actual capacitive couplings between graphene and source and drain electrodes. Modeling this relation is beyond the scope of this work. ${ }^{16}$ Nevertheless, for very long channels where the source and drain electrodes are far away from each other and ignoring band bending at the contacts, an approximate analytic expression between $E_{F}$ and $V_{G}$ can be obtained, ${ }^{11}$

$$
e V_{G}=\frac{e^{2} d}{\epsilon} \frac{16 \beta}{3 t^{2} a^{2}} E_{F}^{2}+E_{F},
$$

where $a$ is the graphene lattice constant and $\beta$ is a dimensionless parameter $(\leq 1)$ which accounts for the finite width of the channel ${ }^{11}$ (bulk graphene corresponds to $\beta=1$ ). A realistic situation that reduces the classical electrostatic capacitance corresponds to, e.g., $d=7 \mathrm{~nm}$ and $\epsilon=47$. Based on selfconsistent calculations using the same methodology presented in Ref. 11 , we estimate $\beta$ to be in the range of $\approx 0.1-0.2$ for the channel widths considered.

\section{ROLE OF METAL CONTACTS}

Finally, to better model a real experimental situation, we consider the role played by the source and drain metallic electrodes which are needed to connect the ribbon to the external circuitry. At room temperature, when the metallic electrodes are sufficiently far from the constriction, electrons may loose phase coherence before reaching it. In this limit, we can consider the total conductance to be given by

$$
G_{T}=\left(R_{C}+R_{M}^{S}+R_{M}^{D}+R_{Q}\right)^{-1},
$$

where $R_{C}$ is the so-called four-terminal resistance of the constriction, and $R_{M}^{S}$ and $R_{M}^{D}$ are the four-terminal resistances (hereon considered equal) due to the contact with the source and drain metal electrode, respectively,

$$
R_{C(M)}=\frac{h}{2 e^{2}} \frac{N-T_{C(M)}}{N T_{C(M)}} .
$$

A new transmission function, $T_{M}\left(E_{F}\right) \leq N\left(E_{F}\right)$, has been introduced to account for the scattering at the interface between the metal and ribbon. Finally, $R_{Q}=\frac{h}{2 e^{2}} \frac{1}{N}$ is the intrinsic quantum resistance of the ribbon. In this approximation, the total conductance is given by

$$
G_{T}=\frac{2 e^{2}}{h}\left(\frac{2}{T_{M}}+\frac{1}{T_{C}}-\frac{2}{N}\right)^{-1} .
$$

From Eq. (4), one can see that, under the initial assumption $T_{C} \ll T_{M} \leq N$, the overall conductance is determined by the bare value $T_{C}$ calculated in previous sections. On the other hand, in the absence of constriction and scattering at the metal-ribbon interfaces, one recovers the perfect conductance of the ribbon (see inset in Fig. 4). The function $T_{M}$ is, unfortunately, hard to determine and is expected to be strongly dependent on the metal used. Experiments ${ }^{1-4}$ as well as first-principles calculations of this quantity for carbon nanotubes ${ }^{17,18}$ have revealed that $T_{M} / N$ can range from 
$10^{-3}$ to 1 . For Pd electrodes, which have proved to make excellent contacts, $T_{M} / N \approx 0.1-1 .^{2,18}$ For other metals, this factor can be reduced in orders of magnitude.

To illustrate the effect of the metal on the performance of the GRFET, we consider an electrode model in the form of a bidimensional square lattice with the same hopping parameter $t$ as the graphene lattice and varying on-site energy $\epsilon$. The inset in Fig. 4 shows the conductance of a single metalribbon interface for various values of $\epsilon$. As an illustration, Fig. 4 shows $G_{T}$ for the square constriction using $\epsilon=5 t$. The performance of the GRFET's is noticeably affected by the metal electrodes since $T_{M} \approx T_{C} \approx 1$ at $E_{F} \sim e V_{\text {th }}$ in all cases. Scattering at the metal contact will turn, however, irrelevant as $W_{C} / W_{L} \rightarrow 0$. In this limit, while $T_{C}$ remains bound to $<1$ at $E_{F} \sim e V_{\text {th }}, N$ and $T_{M}$ will generically increase because $e V_{\text {th }}$ increases (see inset in Fig. 4). However, as $W_{C} / W_{L} \ll 1$, scattering at the constriction increases. In summary, for $W_{C} / W_{L} \lesssim 1$, scattering will be dominated by the metal contacts, while for $W_{C} / W_{L} \ll 1$, it will be dominated by the constriction. One can thus conclude that an optimal ratio $W_{C} / W_{L}$ that maximizes the conductance of GRFET's at $E_{F} \sim e V_{\text {th }}$ will always exist. This ratio will be nonuniversal and will depend on both the metal contact and the atomic details of the constriction.

\section{CONCLUSIONS}

When compared to bidimensional nonrelativistic electrons, ${ }^{19}$ the scattering due a constriction is much stronger in the case of relativistic ones. This prevents conductance quantization from appearing in these devices. For tapered constrictions, deviations from conductance quantization can still be of the order of $2 \%$ or $3 \%$, while they are of the order of $0.0001 \%$ for nonrelativistic electrons. ${ }^{19}$ Our results support recent claims by Katsnelson, ${ }^{20}$ although more work is needed here to clarify these fundamental issues. When the channel is placed symmetrically in the middle of the ribbon, the scattering turns out to be larger, strongly worsening conductance quantization and the performance of the GRFET. A detailed account of this and other possible contact models that can be considered will be published elsewhere. ${ }^{21}$

Concerning disorder effects, a caveat should be issued. The use of graphene ribbons for field-effect transistors could be hampered by the fact that, in average, one out of three constrictions are metallic, given the unlikely chance that lithography can define the constriction with atomic precision. The same uncertainty in the lithographic definition of the constriction width, with the concomitant disorder at the edge, could, however, open up a gap, transforming otherwise metallic ribbons into a semiconducting ones. ${ }^{22}$

In summary, our results show, generically, that the conductance of graphene ribbons with constrictions in the form of a narrow channel presents a steplike behavior as a function of $E_{F}$ or $V_{G}$. However, one can hardly claim conductance quantization (in units of $2 e^{2} / h$ ) in most cases. At zero temperature, Fabry-Pérot oscillations appear on top of the steps, being more pronounced for the square constrictions. Such oscillations result from finite scattering at the interfaces, which is reduced for the tapered ones, but not completely absent as naively expected. At room temperature, the Fabry-Pérot oscillations disappear even for very short channels of $\approx 10 \mathrm{~nm}$ with abrupt contacts to the bulk ribbon. Due to the relativistic nature of electrons in graphene, $G_{\text {on }}$ does not saturate to the quantum limit $2 e^{2} / h$ close to $V_{\text {th }}$ for neither type of constrictions considered. However, from an operational point of view and compared to making direct contact to metal electrodes, one could say that tapered contacts between a wide graphene ribbon and a narrow graphene channel can perform nearly as Ohmic contacts for intermediate channel widths.

\section{ACKNOWLEDGMENTS}

F.M.R. is indebted to D. Jacob for his help with the transport code and acknowledges the University of Alicante for financial support. We acknowledge F. Guinea and P. Jarillo-Herrero for discussions. This work has been funded by Spanish MEC under Grant Nos. FIS2004-02356, MAT2006-03741, MAT2007-65487, and CONSOLIDER No. CSD2007-00010, by Generalitat Valenciana under Grant No. ACOMP07/054, and by FEDER Funds.
${ }^{1}$ S. Heinze, J. Tersoff, R. Martel, V. Derycke, J. Appenzeller, and P. Avouris, Phys. Rev. Lett. 89, 106801 (2002).

${ }^{2}$ A. Javey, J. Guo, Q. Wang, M. Lundstrom, and H. Dai, Nature (London) 424, 654 (2003).

${ }^{3}$ M. H. Yang, K. B. K. Teo, W. I. Milne, and D. G. Hasko, Appl. Phys. Lett. 87, 253116 (2005).

${ }^{4}$ Z. Chen, J. Appenzeller, J. Knoch, Y.-M. Lin, and P. Avouris, Nano Lett. 5, 1497 (2005).

${ }^{5}$ A. Javey, J. Guo, D. Farmer, Q. Wang, D. Wang, R. Gordon, M. Lundstrom, and H. Dai, Nano Lett. 4, 447 (2004).

${ }^{6}$ K. Nakada, M. Fujita, G. Dresselhaus, and M. S. Dresselhaus, Phys. Rev. B 54, 17954 (1996).

${ }^{7}$ L. Brey and H. A. Fertig, Phys. Rev. B 73, 235411 (2006).

${ }^{8}$ M. Y. Han, B. Ozyilmaz, Y. Zhang, and P. Kim, Phys. Rev. Lett. 98, 206805 (2007).
${ }^{9}$ B. Özyilmaz, P. Jarillo-Herrero, D. Efetov, D. A. Abanin, L. S. Levitov, and P. Kim, Phys. Rev. Lett. 99, 166804 (2007).

${ }^{10}$ Y.-W. Son, M. L. Cohen, and S. G. Louie, Phys. Rev. Lett. 97, 216803 (2006).

${ }^{11}$ J. Fernández-Rossier, J. J. Palacios, and L. Brey, Phys. Rev. B 75, 205441 (2007).

${ }^{12}$ M. Fujita, K. Wakabayashi, K. Nakada, and K. Kusakabe, J. Phys. Soc. Jpn. 65, 1920 (1996).

${ }^{13}$ F. Muñoz-Rojas, D. Jacob, J. Fernández-Rossier, and J. J. Palacios, Phys. Rev. B 74, 195417 (2006).

${ }^{14}$ C. Caroli, R. Combescot, and P. Dederichs, J. Phys. C 4, 916 (1971).

${ }^{15}$ M. Bockrath, D. H. Cobden, J. Lu, A. G. Rinzler, R. E. Smalley, L. Balents, and P. L. McEuen, Nature (London) 397, 598 (1999). 
${ }^{16}$ G. Liang, N. Neophytou, M. S. Lundstrom, and D. E. Nikonov, arXiv:0705.0556 (unpublished).

${ }^{17}$ J. J. Palacios, A. J. Pérez-Jiménez, E. Louis, E. SanFabián, and J. A. Vergés, Phys. Rev. Lett. 90, 106801 (2003).

${ }^{18}$ J. J. Palacios, P. Tarakeshwar, and D. M. Kim, arXiv:0705.1328 (unpublished).
${ }^{19}$ A. Szafer and A. D. Stone, Phys. Rev. Lett. 62, 300 (1989).

${ }^{20}$ M. I. Katsnelson, Eur. Phys. J. B 57, 225 (2007).

${ }^{21}$ F. Muñoz Rojas, J. Fernández-Rossier, and J. J. Palacios (unpublished).

${ }^{22}$ F. Sols, F. Guinea, and A. Castro-Neto, Phys. Rev. Lett. 99, 166803 (2007). 